XXIII. Kongress der Deutschen Gesellschaft für Philosophie 28. September - 2. Oktober 2014, Münster

Sektion Philosophie der Lebenswissenschaften

\title{
What are mental disorders?
}

\section{Patrice Soom}

Münstersches Informations- und Archivsystem multimedialer Inhalte (MIAMI)

URN: urn:nbn:de:hbz:6-12319421845 


\title{
What are mental disorders? ${ }^{1}$
}

\author{
Patrice Soom \\ patrice.soom@phil.hhu.de \\ Heinrich-Heine Universität Düsseldorf \\ Sektion: Philosophie der Lebenswissenschaften
}

02.10 .2014

\section{Abstract}

Mental disorders are currently defined in the official classification systems (DSM-5 and ICD-10) as syndromes consisting in clusters of symptoms combined with various exclusions conditions. However, these systems also assume that the symptoms occurs because of some underlying causes, which explain as why we regularly encounter in the population some specific clusters of symptoms rather than random distribution of symptoms. This paper aims at investigating the ontological status of mental disorders, reviewing the ontological commitments of various theoretical accounts defining mental disorders. It argues that, especially given the current state of the art in empirical research, mental disorders should be conceived as theoretical entities.

\section{The DSM-5 approach of mental disorders}

Introduced with the publication of the DSM-III, the symptom-based approach characterizing both the DSM-5 and the ICD-10 classifications systems constitutes the outcome of the legitimacy crisis of psychiatry within the third quarter of the $X X^{\text {th }}$ century. The third revision of the DSM aimed at responding to the strong criticisms and suspicions addressed to the post-world-war-II American psychiatry by endorsing a strictly atheoretical, purely descriptive and as objective as possible approach to mental illness (Mayes \& Horwitz, 2005). Committing psychiatry to the scientific and deontological standards of evidence-based western medicine, it reaffirmed the status of psychiatry as a sub-discipline of medicine, giving rise to the medical model of psychiatry (Murphy, 2005). Its main features are that i) mental disorders are to be conceived along the case of medical diseases in general, because psychopathology "represents the manifestations of disturbed functions within a part of the body" (Guze 1992, 44). Research and therapeutic strategies with respect to mental illness requires ii) "the consistent application, in psychiatry, of modern medical thinking and methods" (Black 2005, 3). Furthermore, iii) the classification of mental disorders is value-free (see especially Boorse 1975, 1976, 1977, 1997), at least in some minimal sense (Wakefield, 1992).

This approach, of which the DSM-5 is nothing but the direct continuation, defines mental disorders as clinically significant sets of co-occurring behavioral and psychological symptoms combined with exclusion conditions, which facilitate differential diagnosis. More precisely, a mental disorder is defined as "a clinically significant behavioral or psychological syndrome or pattern that occurs in an

\footnotetext{
${ }^{1}$ This paper was presented at the XXIII. Kongress der Deutschen Gesellschaft für Philosophie 2014 in Münster, in the section Philosophie der Lebenswissenschaften, on october the $2^{\text {nd, }} 2014$.
} 
individual and that is typically associated with either a painful (distress) or impairment in one or more important areas of functioning (disability)" (APA, 1980, p. 6). This approach is said "atheoretical" or "descriptive" because it makes no assumption with respect to the causes of disorders. In particular, it is not the case that the causes of the symptoms defining a given disorder are a part of the individuation conditions of that disorder. This syndromal approach persisted in the fourth and fifth editions of the DSM, where a mental disorder is defined as "a syndrome characterized by a clinically significant disturbance in an individual's cognition, emotion regulation, or behavior that reflects a dysfunction in the psychological, biological, or developmental processes underlying mental functioning (APA, 2013, p. 20).

This characterization reveals a deep ambiguity at the core of the medical model of psychiatry. Mental disorders are syndromes, i.e. as clusters of symptoms. It is assumed that these symptoms "reflects" a hypothetical internal dysfunction, which, whatever it is, is supposed to act as a common cause bringing about the observable symptoms. Such dysfunctions are thereby supposed to explain why we encounter stable clusters of symptoms in the population rather than a purely random distribution of symptoms: what holds symptoms together are dysfunctioning latent causes. However, the dysfunctions in question are not a part of the individuation conditions of mental disorders. This approach contrasts with the concept of a medical disease, which refers directly to impaired biological functions causing various symptoms. Here is a way of noticing the difference. In the case of general medical conditions, the relation between disease and symptom is contingent: one might have a disease without manifesting the symptoms, as for instance in incubation stages. This is precisely not the case within the DSM syndromal approach, which takes the relation between disorders and symptoms to be analytic, i.e. one cannot have a given disorder without manifesting the relevant symptoms with a minimal severity and vice-versa.

The guiding question of this paper is to determine if, and if so, how, the medical model of psychiatry might be extended to match the requirements of the model of general medical diseases, which amounts to the question as to whether mental disorders can be defined on the basis of the symptom causes rather than on the basis of symptoms only. The dialectic of this paper shall be as follow. Providing an answer to these questions requires characterizing the dysfunctions causing the symptoms. After shortly summarizing the criticism addressed to the DSM syndromal approach (section 2), I shall assume as a working hypothesis that mental disorders are causes of symptoms rather than clusters of symptoms and review the main theoretical alternatives with regards to the ontological status of mental disorders (section 3). Indeed, dysfunctions require to be characterized against the background of a theory stating what normal functions are. Given the difficulties raised by each of these alternatives, I suggest in the positive part of this paper that mental disorders should be handled as theoretical entities and psychiatric diagnostic categories as theoretical terms (section 4). I shall finally clarify how such an account might handle the objection that is already addressed to the DSM syndromal approach, according to which it faces the risk of lumping together under the same diagnosis categories conditions that are notoriously heterogeneous from a neurobiological point of view (section 5 and 6).

This paper shall not discuss the fundamentally normative question of who is and who is not mentally ill. As such, it does not aim at illumining questions as for instance why certain sets of behavioral traits count as sets of symptoms or why a specific degree of performance in the execution of a given cognitive function counts as dysfunction. It rather focuses on the ontological status of mental 
disorders, highlighting the difficulties raised by the available options when on assume that mental disorders are latent causes of symptoms rather than symptoms clusters simpliciter.

\section{The DSM syndromal approach}

As already mentioned, the DSM-III, -IV, and -5 approaches of mental disorders focuses on symptoms in order to define mental disorders. Mental disorders essentially consist in clusters of symptoms. There is nothing more to the disorder than the symptom presentation, to the extent that a minimal threshold of distress or disturbance in the patient's functioning is reached. Consider for instance Delusional disorders (table 1).

\begin{tabular}{|l|l|}
\hline \multicolumn{2}{|l|}{ Table 1 - Diagnostic criteria for 297.1 Delusional Disorder (APA, 2013, p. 90): } \\
\hline $\begin{array}{l}\text { Criterion } \\
\text { A }\end{array}$ & $\begin{array}{l}\text { The presence of one (or more) delusions with a duration of 1 month or } \\
\text { longer. }\end{array}$ \\
\hline $\begin{array}{l}\text { Criterion } \\
\text { B }\end{array}$ & $\begin{array}{l}\text { Criterion A for Schizophrenia has never been met. Note: if present, } \\
\text { symptoms are not prominent and are related to the delusional theme (e.g., } \\
\text { the sensation of being infested with insects associated with delusion of } \\
\text { infestation). }\end{array}$ \\
\hline $\begin{array}{l}\text { Criterion } \\
\text { C }\end{array}$ & $\begin{array}{l}\text { Apart from the impact of the delusion(s) or its ramifications, functioning is } \\
\text { not markedly impaired and behavior is not obviously bizarre or odd. }\end{array}$ \\
\hline $\begin{array}{l}\text { Criterion } \\
\text { D }\end{array}$ & $\begin{array}{l}\text { If manic or major depressive episodes have occurred, they have been brief } \\
\text { relative to the duration of the delusional disorder. }\end{array}$ \\
\hline $\begin{array}{l}\text { Criterion } \\
\text { E }\end{array}$ & $\begin{array}{l}\text { The disturbance is not attributable to the physiological effects of a } \\
\text { substance or another medical condition and is not better explained in terms } \\
\text { of another mental disorder, such as body dysmorphic disorder or obsessive- } \\
\text { compulsive disorder. }\end{array}$ \\
\hline
\end{tabular}

Accordingly, the presence of the one or more delusions over a period of at least one month (criterion A), combined with the satisfaction of some exclusion criteria (criteria B-E) is sufficient for the ascription of the diagnosis of Delusional disorder. Exclusion criteria B-D aim at delineating precise sets of symptoms by ruling out symptoms, of which the presence would modify the diagnosis. Hence, they do not impinge on the syndromal character of the approach. Criterion $\mathrm{E}$ is more ambiguous. The latter part is innocuous, since Body dysmorphic disorder and Obsessive-compulsive disorder are individuated as different clusters of symptoms. The former part makes reference to non-behavioral and non-mental parameters. This does however not contradict the symptom-based character of the approach, since the satisfaction of these conditions shifts the diagnosis towards a non-psychiatric diagnostic category. If the former condition in criterion $E$ is satisfied, any psychiatric diagnosis is excluded, with the result that no mental disorder is defined by reference to non-symptomal criteria. 
The DSM syndromal approach has been heavily criticized over the last decades. While the public and political discussions focused on the alleged arbitrary and over-inclusive character of diagnostic criteria, some theoretical concerns have also been raised, including, amongst others, the objective character of psychiatric classifications, the use of disjunctive taxa, the mental character of psychiatric conditions, etc. In order to keep our discussion as focused as possible, I shall focus on the syndromal character of the approach, which raises two main concerns. First, it seems to be a general desideratum of scientific activity and explanation to focus on causal explanations (Esfeld \& Sachse, 2011), amongst other reasons because, as nicely put forward by Woodward, causal relations are these relations that are exploitable for purpose of manipulation and control (Woodward, 2003). Obviously, defining mental disorders as clusters of symptoms does not, per se, prevents the development of various types of causal accounts with regards to the symptoms production, evolution or responses to treatment. However, it puts some distance between the theoretical foundations of psychiatry and the general medical model of diseases, where research mostly focuses not on the symptoms, but on their causes. Medical treatments typically target the disease itself, i.e. the causes of the symptoms, rather than the symptoms.

Second, and most importantly for our purposes, the DSM approach faces the risk of lumping together medical conditions that are potentially extremely heterogeneous from a neurological perspective. The objection is rooted in the observation that biological systems display an important degree of variability at the individual level (Boyd, 1999; Wilson, Barker, \& Bringandt, 2007). This implies that a given type of mental disorder might arise as the outcome of very different neurobiological pathomechanisms. In a nutshell, the fact that psychiatric categories focus on symptoms undermines empirical research because it fails to put the emphasis on the causal structure at work in the symptom production. Arguably, focusing on the causal structures leading to the symptoms paves the way to optimal explanations, course predictions and response to treatments. By contrast, if psychiatric categories focusing on symptoms lump together heterogeneous neurobiological conditions, then diagnoses based on these categories cannot distinguish between different forms of neurobiological dysfunctions, each of which is susceptible to have a specific course and its own response to treatment. Thereby, overlooking causal structures, the DSM syndomal approach directly undermines our ability to make accurate predictions.

\section{Scientific essentialism}

These elements motivate a stronger interpretation of the medical model of psychiatry, where, mental disorders essentially consist in the pathological dysfunctionning of the mechanisms that are causally responsible for the symptoms occurrence. Accordingly mental disorders are pathological dysfunctions of cognitive systems. Let us label this kind of position "strong essentialism" given its obvious connection with scientific essentialism (see for instance Bird, 2007; Ellis, 2001). Note that, in addition to the aforementioned elements, an appealing feature of strong essentialism is that it promises to confer to psychiatric diagnosis some degree of objectivity because it aims at defining mental disorders as abnormal alteration of specific psychological, cognitive, neurobiological functions, which are themselves defined against the background of well-established scientific theories. 
The difficulties for strong essentialism start when one asks what the theory is, against the background of which the dysfunctions in question should be characterized. This question is important, since strong essentialism amounts to redefine mental disorders against the background of a specific scientific theory. Let us start with some theorists favoring strongly physicalist views here. The clearest example of such a strongly reductive framework is certainly Kandel's New Intellectual Framework for Psychiatry (1998), where the correct level of scientific theorization in psychiatry is held to be genetic neurobiology. The reason is that gene expression constitutes the interface between properly genetic predispositions and environmental influences. Gene expression modifications are involved in many medical condition, but also in cognitive processes as long-lasting learning (LTP), and thus, crucially, in the development of psychiatric conditions. Emphasizing that psychotherapy and pharmacotherapy also impact on the patient's condition by durably modifying patterns of synaptic connectivity, and thereby also modify the gene expression, the account suggests that mental illness should be characterized at this level of analysis. Kandel's framework constitutes a perfect example of strong scientific essentialism: mental disorders are adequately characterized as abnormal neurobiological conditions, which might be due either to purely genetic factors or to the interaction of genetic predispositions and environmental factors.

Kandel's program might be interpreted either as an attempt to reduce the current classification to some neurobiological classification or as a way of eliminating the current classification to the benefit of a totally new classification based on neurbiological criteria. Each interpretation raises considerable difficulties. The first one requires nomologically necessary bi-conditional bridge-principles between our current disorder categories and categories constructed using the vocabulary of the reducing theory. As a result, strong essentialism implies that any individual who does not satisfy the target condition $N$ in the reducing theory cannot be said to have mental disorder $M$, even though he or she manifests all the symptoms of $M$. The obvious worry is that the above mentioned important degree of variability observed in biological and biomedical sciences suggests that, at least for some mental disorders, several underlying neurobiological conditions exist. Indeed, just as mental properties are said to be multiply realizable, so are mental disorders: to the extent that mental disorders are individuated on the basis of symptoms, it is perfectly possible that neurobiologically different systems produce the same set of symptoms. This interpretation of Kandel's project is therefore heavily compromised.

The second interpretation of Kandel's program consists in discarding completely the current classification and redefining mental disorders entirely on the basis of neurobiological criteria, without any reference to behavioral and psychological symptoms or to the current classification. Some questions arise in this case as well. First, given that psychiatric categories are individuated on neurobiological grounds, patients have to be diagnosed based on neurobiological criteria only. Therefore, there is no scientific ground for grouping together patients on the basis of psychological and behavioral similarities. The psychological and behavioral commonalities that are at the root of the current classification system would be left with a purely heuristic role, guiding scientists within the process of selecting the sets of individuals on the basis of which neurobiological inquiries might be conducted. Besides, we clearly fly away from the way we usually identify mental illness.

Second, strong essentialism aims at anchoring mental disorders within the causal structure responsible for the occurrence of the symptoms, in order to improve our ability to make course prediction and to develop therapeutic interventions. Given the underlying variability challenge to the 
DSM approach, we might expect that such an approach will be developed into a system of classification that is finer-grained and where the extension of each category is smaller than in the actual system. Admittedly, there is certainly an explanatory and pragmatic interest at having the most detailed descriptions and predictions. Note however that we gain the ability to make better predictions that are specific to smaller sets of patients to the extent that we lose the ability to make coarser-grained predictions that apply to larger sets of patients. We face here a trade-off rather than a win-win situation: Arguably, discarding the current taxonomy to the benefice of a neurobiological taxonomy yields the risks of losing explanatorily interesting generalizations concerning patients presenting salient behavioral and psychological similarities.

Finally, let us stress a pragmatic point. Re-defining mental illnesses in terms of neurobiological abnormalities is at best a very long term project and at worst impossible. Neurobiological psychiatry is by far not ready yet to take the highly multidisciplinary scientific aspects and the clinical practice of psychiatry over. A strict interpretation of Kandel's program requires the reformulation of our psychiatric knowledge in some neurobiological terms. The obstacle here consists in the fact that an important part of this knowledge concerns psychological and behavioral facts. To offer a simple example: it is unclear how to grasp in neurobiological terms the generalization formulated by Kendler and Precott (2006, p. 148), that stressful life events with humiliating features are highly depressogenic.

Strong essentialism does not have to rely on neurobiology. The theory against the background of which the essence of the mental disorder might be characterized might be a higher-level theory, such as cognitive psychology. Heinrich (2001) and Andreasen (1997) argued that the correct framework in order to describe the dysfunctioning mechanisms in which psychiatry trades is cognitive neuroscience broadly construed. Indeed they draw a theoretical picture that fits very well the widespread idea of functional analysis (Cummins, 1975, 2000) or mechanistic explanations (Craver, 2007). Their account constitutes a strong interpretation of the medical model in the sense that "contemporary psychiatry [...] is the discipline within cognitive neuroscience that integrates information from all these related disciplines in order to develop models that explain the cognitive dysfunctions of psychiatric patients based on knowledge of normal brain/mind function" (Andreasen, 1997, p. 1586).

While there is certainly a significant interest in bringing psychiatry and contemporary cognitive neuroscience closer, it is first unclear if cognitive neuroscience currently is an established theory with a list of admitted principles that can ground the classification of mental disorders. The individuation of cognitive functions is highly indirect and still subject to important debates. Furthermore, the complex interaction between different cognitive functions unfolding from the alteration of one of them is poorly understood. In short, the relations between cognitive functions and complex patterns of symptoms are poorly understood.

Besides, it is unclear from a theoretical perspective as to whether cognitive neuroscience constitutes a unified theory with unified explanatory principles, since it combines data acquired using very different methods and originating from different fields and levels of explanation. Furthermore, while Andreasen's explanatory scheme seems close to what philosophers call functional analysis or reductive explanations, it is unclear what the detail of the adequacy conditions for these explanations are. With respect to these issues, Kendler, Zachar and Craver forward the proposal that 
mental disorders consist in clusters of impaired mechanistic properties, "which are themselves fuzzy sets defined by mechanisms at multiple levels that act and interact to produce the key features [of the disorder kind]" (K. S. Kendler, Zachar, \& Craver, 2011). This account relies on Craver's mechanistic account of the unity of neuroscience (2007). Accordingly, cognitive neuroscience, including psychiatry when it comes to cognitive impairments, essentially aims at explaining higher-level phenomena by means of lower-level phenomena. The central idea is that a mechanism $m$ for the phenomenon $p$ is a collection of $\delta$-organized components $c_{1}, \ldots c_{\mathrm{m}}$ performing the activities $\phi_{1}, \ldots, \phi_{\mathrm{m}}$ such that $m$ as a whole exhibits the causal role $\psi$ of $p$ (Craver, 2007, pp. 6-7). The unity of cognitive neuroscience is achieved by gathering explanatory resources in different sub-disciplines in order to show how a given cognitive phenomenon is integrated into a nested hierarchy of mechanisms both as a component of a mechanism explaining a higher-level phenomenon, and as a phenomenon that is itself explained by a lower-level mechanism (Craver, 2007, pp. 257-258).

Heinrich's, Andeasen's and Kendler and collegues' accounts share the central idea that contemporary psychiatry should be grounded within contemporary cognitive neuroscience, which is an intrinsically multi-level discipline explaining cognitive phenomena reductively by means of lower-level phenomena, realizers or mechanisms. While it is indisputable that contemporary psychiatry does actually refer to variables located at different levels, relating ontologically heterogeneous variables does not come without difficulties. To cut a long story short, the classical problem arising is the wellknown causal exclusion problem (Kim, 2005), which stresses the tension between the claims i) that higher-level entities can causally influence lower-level phenomena and ii) that the physical world is causally close and complete. In short, to the extent that the physical world is causally complete and closed, it is unclear how higher-level variables might explain anything, since every aspect of the behavior, including psychiatric behavioral symptoms, clearly has a physical complete and sufficient cause. Besides, Craver's mechanistic account also faces important metaphysical difficulties, which are related to the fact that he relies on interventionism in order to account for inter-level relations (Baumgartner, 2009). The worry here is that interventionism requires the Causal Markov condition to be satisfied, whereas inter-level composition relations are typically non-causal determination relations, excluding thereby the satisfaction of the Causal Markov condition (Soom, 2012).

Finally, one might favor a purely psychological version of strong essentialism, where the essence of mental disorders lays in some faulty psychological process. There are at least two immediate difficulties. First, common-sense psychology cannot play the central role here, since a mental illness precisely appears that stunning because it hurts one's common-sense interpretation of other agents' behavior. The debate with respect to the doxastic status of delusions is revealing: delusions seem not to be belief-type mental properties, since they clearly violate the standard requirements for belief ascription (Bortolotti, 2010). While psychoanalysis might be a candidate, it is unclear if it covers the entire spectrum of what we considers as mental illnesses and whether if it does - or can characterize these within a theoretical framework that fits contemporary scientific standards (Lacewing, forthcoming). Finally, the central problem of a version of scientific essentialism relying on some psychological theory is the same as the one affecting the DSM descriptive approach in the first place: focusing on abstract categories leads to neglecting the underlying causal structures, which might turn out to be very heterogeneous. The lumping charge is back on stage. Furthermore, it would also be problematic to account for how these higher-level purely mental disorders would bring about symptoms that are themselves behavioral and therefore physical: the causal exclusion problem kicks in again. 


\section{Mental disorders as functional theoretical entities}

The positive proposal of this paper is that mental disorders should be conceptualized as theoretical entities that are extrinsically individuated by their typical causal relations to symptoms. Accordingly, anything causing the relevant sets of co-occurring symptoms qualifies as an instance of a given mental disorder type. Mental disorders are then theoretical dispositions to cause specific sets of symptoms. Just as the current DSM-approach, this account individuates mental disorders on the basis of the symptoms they produce. It however enforces a stronger version of the medical model by discarding the syndromal character of the DSM approach, since mental disorders are causes of symptoms rather than symptoms clusters simpliciter. Let us label this proposal "dispositional essentialism".

Consider once again the case of Delusional disorder. DSM-5 diagnostic criteria mention positive symptoms and exclusion conditions. Within dispositional essentialism, criterion $A$ should be interpreted as specifying that delusional disorders cause delusions. Criterion B reduces to the nonproduction of other symptoms, which might be grasped functionally by specifying that delusional disorders do not cause delusions that are bizarre, hallucination, disorganized speech, grossly disorganized or catatonic behavior, affective flattening, alogia or avolitions. Criterion $\mathrm{C}$ specifies that no other behavioral specificity is present, and might thereby be set aside. Criterion D and E excludes the presence of other symptoms and/or causes and thereby rules out alternative diagnoses.

A generic definition of theoretical terms might be provided by the standard method of ramsification. Definition $F$ specifies that an individual $y$ has disorder $\Phi$ if and only if i) $y$ has the relevant sets of behavioral and psychological symptoms, ii) $y$ does not satisfy the conditions for some other diagnoses and iii) the symptoms are caused by the disorder. Condition i) grasps the symptoms, usually described in the section A of DSM diagnostic criteria.

(F) $y$ has mental disorder $\Phi$ if and only if $\left(\exists x_{1}\right)\left(\exists x_{2}\right)\left(\exists x_{3}\right) \ldots\left(\exists x_{j}\right)[T(y$ has symptoms $\left.b_{1} \wedge b_{2} \wedge \ldots \wedge b_{\mathrm{m}}\right) \wedge\left(y\right.$ does not have symptoms $\left.b_{\mathrm{n}} \wedge \ldots \wedge b_{\mathrm{q}}\right) \wedge(y$ has not $\left.x_{2} \wedge x_{3} \wedge \ldots \wedge x_{\mathrm{j}}\right) \wedge\left(x_{1}\right.$ causes symptoms $\left.b_{1} \wedge b_{2} \wedge \ldots \wedge b_{m}\right) \wedge\left(y\right.$ has $\left.\left.x_{1}\right)\right]$.

Given the general character of the DSM approach, an analog definition might be constructed for any mental disorder. Therefore, the disorders represented by variables $x_{2}, \ldots, x_{n}$ might also be replaced by relevant sets of symptoms, with the result that any mental disorder might be represented as a disposition to cause a specific set of symptoms, without reference to further mental disorders. It is therefore in principle possible to build, for any disorder, a definition mentioning only symptoms that have to be either specifically present or absent, delineating thereby precise sets of symptoms. The generic definition $\mathrm{F}^{*}$ grasps the core of dispositional essentialism, i.e. that mental disorders are dispositions possessed by patients to cause specific sets of symptoms.

$\left(\mathrm{F}^{*}\right) y$ has mental disorder $\Phi$ if and only if $\left(\exists x_{1}\right)\left[T\left(y\right.\right.$ has symptoms $b_{1} \wedge b_{2} \wedge \ldots \wedge$

$\left.b_{\mathrm{m}}\right) \wedge\left(y\right.$ does not have symptoms $\left.b_{\mathrm{n}} \wedge \ldots \wedge b_{\mathrm{q}}\right) \wedge\left(y\right.$ has $\left.x_{1}\right) \wedge\left(x_{1}\right.$ causes symptoms $\left.\left.b_{1} \wedge b_{2} \wedge \ldots \wedge b_{\mathrm{n}}\right)\right]$. 
Let us emphasize the following elements. First, it is possible to integrate diachronic etiological causes of mental disorders to this approach. Such a functional definition might always be further specified by adding further constraints, as long as these constraints are causally interpretable. For instance, Kendler \& Prescott argues as mentioned that "stressful life events" are among the chief causes of depression, especially if they involve humiliation experiences. If required, such elements might be incorporated into an extended functional specification by adding a further element specifying typical causes.

Second, a dispositional approach of mental disorders constitutes a starting point towards the reductive investigation of the neurophysiological impairments underlying mental disorders. Unless one redefines mental disorder entirely in neurobiological terms, one needs to operationalize mental disorders in such a way that it provides validation conditions for subsequent reductive inquiries. Stepping aside from psychiatry for an instant, consider the very project of providing reductive accounts of cognitive functions like spatial memory, action planning, visual guidance, priming, etc. Most recent theoretical accounts share the idea that higher-level cognitive phenomena have to be functionally defined in the first place. Higher-level cognitive functions, or dysfunctions, are then explained by reference to a given underlying cognitive mechanism (Craver, 2007; Machamer, Darden, \& Craver, 2000), realizer (Chalmers, 1996, pp. 42-51; Kim, 2005, pp. 108-120) or set of sub-functions (Cummins, 1975, 2000) just in case the functions to be explained and the underlying structures are shown to have identical causal roles. The present account aims at bringing psychiatric explanation along these models, by analyzing mental disorders has having a typical causal role, which might be reductively explained in a similar way by reference to lower-level pathomechanisms.

What are then mental disorders? We started by raising the question of the nature of mental disorders and examining different essentialist approaches to mental illness, concluding that mental disorders categories should be individuated as theoretical terms. If psychiatric categories are individuated in such a way, what are then the referents of these terms? Theoretical terms have to be understood as reference fixing devices: for any entity, satisfying the conditions specified in the definition of a given theoretical term is sufficient for the entity to be a case of the category in question. This is the crucial advantage of dispositional essentialism over strong essentialism: while it characterizes mental disorders as causes of the symptoms, it does neither specify at which ontological level mental disorders are located, nor what their intrinsic identity at these levels is.

Let us be more specific. Given the aforementioned difficulties concerning the causal efficacy of higher-level variables with respect to behavior production, we are inclined to assume that mental disorder terms refer to neurobiological abnormalities, of which the causal efficacy is nonproblematic. Provided that dispositional essentialism fixes the reference of theoretical terms extrinsically, i.e. by specifying a network of causal relations, it is fully compatible with the underlying variability encountered in psychiatry: one and the same type of mental disorder might be underlain by different types of neurobiological abnormalities. The crucial benefit is that it is possible to take the referents of psychiatric terms to be physical or neurobiological entities, bypassing thereby the difficulties faced by non-fundamental forms of strong essentialism, while remaining compatible with the fact that individuals having the same type of disorder might nonetheless differ from a neurobiological point of view. 
However, the lumping charge addressed to the DSM-descriptive approach kicks once again in. Dispositional essentialism might well lump together conditions that are highly heterogeneous from a neurological perspective. Construed as dispositions to cause sets of symptoms, mental disorders are abstract theoretical entities and thereby dispositional essentialism ignores the underlying finegrained pathological structures, which in turn undermines our ability both to make course predictions and to develop optimal therapeutically interventions.

\section{Specifying psychiatric sub-types: a walkthrough}

The last sections of this paper provide a way out of this problem based on some recent developments in the philosophy of special sciences. The idea is here to introduce disorder sub-types that are specific to certain neurobiological types of dysfunctions and that thereby provide genuine causal information about the underlying causal structure involved in psychiatric conditions. At the same time, this proposal preserves the idea that mental disorders have to be individuated as dispositions to cause behavioral and psychological symptoms.

To begin with, psychiatric sub-types already constitute an important component of the current classification of mental disorders. The diagnosis of Major depressive disorder, single episode, might for instance be further specified as "with mood-congruent psychotic features", "with catatonia", "with melancholic features", etc (APA, 2013, p. 162). Focusing on the default, the melancholic and the psychotic sub-types of depression, Malhi et al. suggest "that [these] three structural depressive subtypes appear functionally underpinned by differential contributions of serotonergic, noradrenergic and dopaminergic neurotransmitters, so influencing phenotypic distinction (our structural model) and allowing an aetiological model to be derived with treatment specificity implications" (Malhi, Parker, \& Greenwood, 2005, p. 94). Following the model by Parker (2000), they assume at the observational level that all sub-types involve a depressed mood, while melancholic features are superimposed in the second case and further psychotic features are present in the third case. They argue that at the neurophysiological level, "each class involves the perturbation of separate (albeit, at times overlapping) [underlying] components that may undergo progressive recruitment across the classes" (Malhi, et al., 2005, p. 95). The main results are i) that serotonergic dysfunctions account for the shared mood component across the three considered sub-types, ii) that the interaction between serotonergic and noradrenergic dysfunctions underlays melancholic forms of depression, and iii) that psychomotor disturbance and psychotic features of depression results from of a diminished cerebral dopamine regulation.

With respect to the lumping charge addressed to the DSM approach and to dispositional essentialism, the question is as to whether or not some further underlying variability subsists between patients falling under the very same psychiatric sub-type. If it is not the case, then Malhi et al. account provides necessary and sufficient neurophysiological conditions that are systemically correlated with observational sub-types. Observational sub-types and neurophysiological types are therefore co-extensional. Three consequences follow. First, all predictions that can be done on the basis of the description of the underlying neurobiological pathomechanism might by projected onto the corresponding observationally defined sub-type, and vice-versa. Both kinds of types have thus the same predictive value. Second, techniques of therapeutic interventions based on the knowledge of the underlying causal structure might be applied on the basis of the patient's observational 
description. Third, since we assume as our working hypothesis that there is no further variability distinguishing patients falling under the same sub-type, dispositional essentialism cannot be charged of lumping together heterogeneous conditions. As a result, under the working hypothesis that no further underlying variability subsists, an important arguments against dispositional essentialism vanishes.

Let us now turn to the second case, where some further underlying variability between patients falling under the same sub-type is observed. In this case, the underlying variability challenge stands. Our suggestion consists in a generalization of Malhi's et al. observations with respect to depression: whenever different pathomechanisms appear to underlie the same higher-level observationally defined psychiatric type or sub-type, we should construct further refined observational sub-types, such that each one grasps from the psychological and behavioral perspective effects that are specific to each type of neurobiological pathomechanism. From that point of view, the capacity of dispositional essentialism to handle the underlying variability challenge boils down to the question as to whether or not there are different pathomechanisms that always produce, whatever the circumstances are, the same effects and that cannot be distinguished using the descriptive resources of the DSM symptom-based approach.

Taking into account space limitations for this paper, let us offer some brief remarks suggesting that this is not the case. To begin with, contra the initial formulation of the multiple realization argument by Fodor (1974) and Putnam (1967), it is nowadays common grounds in the philosophy of cognitive science that there is limited empirical evidence supporting paradigmatic examples of radical multiple realization. In other words, we still lack of genuine cases in which neurobiological differences do not lead to functional difference at all (Polger, 2008; Shagrir, 1998; Shapiro, 2000; Soom, 2011 section 5.3). Arguably, different mechanisms might produce the same effect to the extent that these effects are only coarsely described. However, whenever one relies on more fine grained descriptions of behavior, one can observe what is called "incidental effects" (Cummins, 2000) or "side effects" (Esfeld, Sachse, \& Soom, 2012). The use of a finer grain of description reveals that different mechanisms produce slightly different effects in behavior (Bechtel \& Mundale, 1999), for instance in reaction time, precision in task execution, etc.

Second, it is precisely a part of cognitive neuroscience methodology and background assumptions that specific experimental protocols might be used in order to magnify behavioral consequences of neurobiological differences (or the converse) and to establish statistically significant double differentiations correlating the behavioral performance of different groups of individuals with their respective neurobiological specificities. The possibility of exploiting specific tasks and experimental designs in order to highlight a double correlation between behavioral and neurobiological specificities is a central assumption and de facto an important element of the methodology of cognitive science (Soom, Sachse, \& Esfeld, 2010). Experimental design plays here a crucial role. Very specific environmental conditions are used in order to observe behavioral differences that would not appear in other, more usual, conditions.

Third, there are even theoretical reasons to assume that neurobiological differences are systematically correlated with observable behavioral differences. However, given that we do not intend to defend the reducibility of mental disorders sub-types to their respective underlying pathomechanisms, the defense of the nomologically necessary character of this claim exceeds the 
scope of the present paper. We refer the readers to Esfled \& Sachse (2011), Shapiro (2000), and Soom (2011 chapt. 6) for further theoretical considerations grounding the argument that, as a matter of nomological necessity, neurological differences imply behavioral differences, to the extent that adequate environmental conditions are provided. The crucial idea here is that two different mechanisms might certainly cause similar effects or symptoms under some specific circumstance, but not under all nomologically possible circumstances.

Let us reframe the bigger picture. Dispositional essentialism defines mental disorders as theoretical entities that might vary in term of compositions and thereby faces the charge of lumping together neurobiological pathological conditions that are possibly heterogeneous. However, provided that refined descriptive resources taking the environmental circumstances are available, a corresponding variability might be observed at the behavioral level with the result that a sub-type of disorder might be specified for each type of pathomechanism. Therefore, it is in principle possible to construct functional sub-types of mental disorders that are co-extensive with neurobiological types of pathomechanisms.

The co-extensionality in question constitutes the crucial step in order to meet the underlying variability challenge. Sub-types of mental disorders do not face this objection, since they do not lump together heterogeneous conditions. Furthermore, i) they implicitly provide information about the actual underlying causal structures, ii) they enable exactly the same set of predictions as the neurobiological descriptions of the corresponding pathomechanisms and iii), given that they convey information about the causal properties of the underlying pathomechanisms, they ground informed therapeutical decisions.

\section{Abstract categories in psychiatry}

The lesson to be drawn from the theoretical possibility of individuating functional sub-types is that the symptom-based individuation of psychiatric categories does not inherently undermine the explanatory and predictive value of psychiatric categories: if the symptom description grain is refined, the extension of types is narrowed down and sub-types highlight refined psychiatric similarities that are shared by relatively small group of patients, with the result that refined predictions are available.

Conversely, neglecting the additional details that are usable in order to build sub-types allows for retrieving DSM categories with larger extensions. Membership to such abstract categories allows for general predictions, because psychiatric taxa are, according to dispositional essentialism, causally defined. However, enlarging the extension of categories by lumping together heterogeneous phenomena has its own price, of which the measure is the fact that these categories provide highly statistical and thereby less specific, predictions. In a nutshell, they provide only coarse-grained predictions.

Do we need such abstract and coarse-grained types? The desideratum of establishing maximally precise and specific predictions is certainly a central goal of science that is at work in contemporary psychiatry. This aim should however screen off the interest of grasping abstract causal similarities between systems that are slightly different. In a certain sense, any actual scientific discipline stands 
on a continuum between physics on the one hand, which aims at formulating strict laws yielding maximally precise predications, and the so-called special sciences on the other hand, which establish weaker predictions for entities that are only imperfectly similar. These ones nonetheless share objective commonalities at the macroscopic level, namely the fact that they exhibit similar behavior under usual circumstances, which grounds their common classification.

Psychiatry occupies an ambivalent position on this continuum. It aims at explaining abnormal mental phenomena as precisely and as accurately alongside the methodology of contemporary cognitive neurosciences. Obviously, this pushes psychiatry towards the redefinition of mental disorders in terms of abnormal underlying pathomechanisms, bringing thereby psychiatry towards the physics side of the continuum. At the same time, the underlying variability encountered in cognitive and life sciences should not screen off important clinically observed commonalities, on the basis of which we identify mental illnesses in the first place. It has to be emphasized that only an abstract classification can highlights similarities that are shared by relatively larger sets of patients and allows formulating general, albeit less precise, predictions. Crucially, there is no principled reason for why precision of prediction should always be preferred over extension size.

Let us finally clarify that it is not our purpose here to formulate any general recommendation with regards to the appropriate degree of precision for in the individuation of mental disorders. This essentially depends on our scientific and therapeutic goals. In certain cases, one might desire having as much detail as possible. As argued, the purely scientific project of bringing psychiatry closer to cognitive science and of accounting for mental disorders in terms of underlying pathomechanisms certainly requires the finest degree of description available. At the same time, this might not be necessary in all cases. This is a matter of conceptual and scientific leeway that should be decided on a case-by-case basis by psychiatrists.

\section{Concluding remarks}

In a theorist's dream, each DSM category would smoothly reduce to a single, necessary and sufficient underlying neurological condition, with the result that our classification of mental disorders might be firmly anchored in a unified neurobiological theory of normal and altered cognition. Unfortunately, there are good reasons to think that such a program cannot be achieved. In particular, the underlying variability encountered in life sciences prevents the establishment of the bi-conditional bridge-laws required for inter-theoretic reduction. The metaphysical puzzle addressed here consists in developing an account of mental disorders, which defines mental disorders as causes of symptoms without taking commitments with respect to their neurobiological or cognitive nature.

We offered an analysis where mental disorders are theoretical entities individuated by the causal relations in which they stand to symptoms. Thereby, a strict interpretation of the medical model is vindicated, since mental disorders are causes of symptoms rather than mere collections of symptoms. At the same time, individuating mental disorders extrinsically avoids any commitment with respect to the ontological status and constitution of mental disorders. That way, it avoids specifying a unique neurobiological condition that corresponds to each mental disorder. The underlying variability observed in the biological sciences cannot here raise worries. At the same time, the possibility of constructing sub-types of mental disorders is the key to avoid the lumping charge 
addressed to the DSM descriptive approach. The theoretical possibility of establishing bi-conditional links between the sub-elements of a symptom-based causal classification and a neurobiological characterization of disorders ensures that the constructed sub-types yield relevant information with respect to the corresponding neurobiological condition. It furthermore secures that the resulting taxonomy might be in principle safely connected with the ongoing research in cognitive neuroscience. Dispositional essentialism thereby preserves a symptom-based approach of mental disorders while securing a close connection to contemporary research in cognitive neuroscience.

\section{References:}

Andreasen, N. C. (1997). Linking Mind and Brain in the Study of Mental Illness: A Project of a Scientific Psychopathology. Science, 275, 1586-1593.

APA (Ed.). (1980). Diagnostic and Statistical Manual of Mental Disorders (DSM-III). Washington DC.

APA (Ed.). (2013). Diagnostic and Statistic Manual of Mental Disorders, Fifth Edition (DSM-V). Arlington: Amercian Psychiatric Association.

Baumgartner, M. (2009). Interventionist Causal Exclusion and Non-reductive Physicalism. International Studies in the Philosophy of Science, 23, 161-178.

Bechtel, W., \& Mundale, J. (1999). Multiple realizability revisited: linking cognitive and neural states. Philosophy of Science, 66(2), 175-207.

Bird, A. (2007). Nature's Metaphysics: Laws and Properties. Oxford: Oxford University Press.

Bortolotti, L. (2010). Delusions and other irrational beliefs. Oxford: Oxford University Press.

Boyd, R. (1999). Homeostasis, species, and higher taxa. In R. A. Wilson (Ed.), Species: New Interdisciplinary Essays. Cambridge, MA: A Bradford Book/MIT Press.

Chalmers, D. J. (1996). The conscious mind. In search of a fundamental theory. New York: Oxford University Press.

Craver, C. F. (2007). Explaining the Brain. Mechanisms and the Mosaic Unity of Neuroscience. Oxford: Clarendon Press.

Cummins, R. (1975). Functional analysis. The Journal of Philosophy(Nov), 20.

Cummins, R. (2000). "How does it work" versus "what are the laws?": Two conceptions of psychological explanation. In F. Keil \& R. A. Wilson (Eds.), Explanations and Cognition. MIT: MIT Press.

Ellis, B. (2001). Scientific essentialism. Cambridge: Cambridge University Press.

Esfeld, M., \& Sachse, C. (2011). Conservative Reductionism. New York: Routledge.

Esfeld, M., Sachse, C., \& Soom, P. (2012). Marrying the merits of Nagelian reduction and functional reduction. Acta Analytica, 27(3), 217-230.

Fodor, J. (1974). Special sciences. Synthese, 28, 97-115.

Heinrich, R. W. (2001). In Search Of Madness; Schizophrenia and Neuroscience. New York: Oxford University Press.

Kandel, E. R. (1998). American Journal of Psychiatry. A New Intellectual Framework for Psychiatry, 155(4), 457-469.

Kendler, K. S., \& Prescott, C. A. (2006). Genes, Environment, and Psychopathology: Understanding the Causes of Psychiatric and Substance Use Disorders. New York: The Guilford Press.

Kendler, K. S., Zachar, P., \& Craver, C. (2011). What kinds of things are psychiatric disorders? Psychological Medicine, 41, 1143-1150.

Kim, J. (2005). Physicalism, or Something Near Enough. Princeton: Princeton University Press.

Lacewing, M. (forthcoming). Could psychoanalysis be a science? . In K. W. M. Fulford, M. Davies, R. Gipps, G. Graham, J. Sadler, G. Stanghellini \& T. Thornton (Eds.), Oxford Handbook of Philosophy and Psychiatry. Oxford: Oxford University Press. 
Machamer, P., Darden, L., \& Craver, C. F. (2000). Thinking about Mechanisms. Philosophy of Science 67, 1-25.

Malhi, G. S., Parker, G. B., \& Greenwood, J. (2005). Structural and functional models of depression: from sub-types to substrates. Acta Psychiatrica Scandinavica, 111, 94-105.

Mayes, R., \& Horwitz, A. (2005). DSM-III amd the revolution in the classification of mental illness. Journal of the History of the Behavioral Sciences, 41(3), 249-267.

Murphy, D. (2005). Psychiatry in the Scientific Image: MIT Press.

Parker, G. (2000). Classifying depression: should paradigms lost be regained? Americal Journal of Psychiatry(157), 1195-1203.

Polger, T. W. (2008). Evaluating the evidence for multiple realization. Synthese, 167, 457-472.

Putnam, H. (1967). The nature of mental states. In Capitan \& Merrill (Eds.), Art, Mind, and Religion: Pittsburgh University Press.

Shagrir, O. (1998). Multiple realization, computation and the taxonomy of psychological states. Synthese, 114, 445-461.

Shapiro, L. (2000). Multiple realizations. Journal of Philosophy, 97, 635-654.

Soom, P. (2011). From Psychology to Neuroscience. A New Reductive Account. Frankfurt (Main): Ontos-Verlag.

Soom, P. (2012). Mechanisms, determination and the metaphysics of neuroscience. Studies in History and Philosophy of Biological and Biomedical Sciences, 43, 655-664.

Soom, P., Sachse, C., \& Esfeld, M. (2010). Psycho-neural reduction through functional sub-types. Journal of Consciousness Studies 17, 7-26.

Wakefield, J. C. (1992). The Concept of Mental Disorder; On the Boundary Between Biological Facts and Social Values. American Psychologist, 47(3), 373-388.

Wilson, R. A., Barker, M. J., \& Bringandt, I. (2007). When traditional essentialism fails: biological natural kinds. Philosophical topics, 35(189-215).

Woodward, J. (2003). Making Things Happen: A Theory of Causal Explanations. Oxford: Oxford University Press. 